\title{
Gekommen, um nicht zu bleiben: Bilder der Ankunft als visuelle Repräsentationen von Migration
}

Schlüsselwörter: Bilder der Ankunft und Abreise, Migration, Gastarbeiter, Auswanderung/Einwanderung, Fotografie

DOI: $10.4312 /$ ars.10.2.31-46

Bilder der Abfahrt und der Ankunft sind eng verknüpft mit Ein- und Auswanderung. Zahlreiche historische Fotografien zeigen beispielsweise die aus Istanbul ab- und in München ankommenden türkischen „Gastarbeiter*innen“ der 1960er Jahre. Bahnhöfe, Züge, Menschen mit Koffern, Unordnung, Neugier und Angst prägen diese dokumentarischen Aufnahmen. Dabei wird das medial verbreitete Bild des überfüllten Bahnhofs zum Topos einer Migration ohne tatsächliche Ankunft in der Gesellschaft - wie auch der Koffer zum repräsentativen Symbol des Einwanderers schlechthin geworden ist. Pendants zu diesen Ankunftsbildern bilden Aufnahmen aus den 1980er Jahren, als türkischen „Gastarbeiter*innen“ hohe Rückkehrprämien geboten wurden. Fotografen wie Brigitte Kraemer oder Manfred Vollmer begleiteten die Familien bei ihrem Auszug aus ihren Wohnungen. Diese nunmehr historischen medialen Szenarien sollen im Beitrag zeitgenössischen Fotografien von Geflüchteten und ihrer Ankunft in Deutschland gegenübergestellt werden: Wie unterscheiden sich die historischen und zeitgenössischen fotografischen Repräsentationen von Einwanderern und Asylsuchenden? Gibt es vielleicht eine übergreifende Bildsprache für den transitiven Zustand der Einwanderung? Welche Zuschreibungen formulieren die Aufnahmen? Und kann die Kunst hier eine Gegenposition einnehmen?

Der Beitrag wird sich mit dokumentarischen, bildjournalistischen und freien künstlerischen Arbeiten zum Thema An- und Abfahrt im Kontext von Migration und Flucht beschäftigen. Die Zusammenführung historischer und zeitgenössischer Materialien soll Aufschluss über Kontinuitäten und Zäsuren im medialen und künstlerischen Umgang mit Einwanderung und Flucht geben. 


\section{Am Bahnhof: Aufbruch und Ankunft von Arbeitsmigrant ${ }^{*}$ innen im Bild}

In ihrem Buch A Seventh Man (Berger, Mohr, 1975) widmeten sich der Fotograf Jean Mohr und der Schriftsteller John Berger den Reisewegen von Arbeitsmigrant*innen nach Westeuropa. Ihre sozialkritische Reportage nahm ihren Ausgangspunkt in den Heimatorten, sie begleiteten die angeworbenen Auswanderer bei entwürdigenden Gesundheitsuntersuchungen in Istanbul (Abb. 1) oder fuhren mit ihnen im Zug nach München. Sie besuchten die Fabriken und Wohnheime, in denen die Arbeiter tätig waren und Unterkunft fanden. Und sie widmeten sich den Besuchen in der alten Heimat. Abschied, Fernweh, Heimweh und Fremdheitserfahrungen liegen in diesem Fotobuch dicht beieinander und werden in literarische Texte und Schwarzweißaufnahmen übersetzt. Dabei ist der Bahnhof ein einprägsames Bild für die Entortung menschlicher Existenz: Mohr fotografierte die Angehörigen (Abb. 2), die an den Gittern Abschied nehmen; die Abfahrt ist hier ein Aufbruch ins Ungewisse, denn vielen Arbeitsmigrant*innen war bis zu ihrer Ankunft am Reiseziel nicht klar, an welchem Ort und für welchen Arbeitgeber sie tätig sein würden.
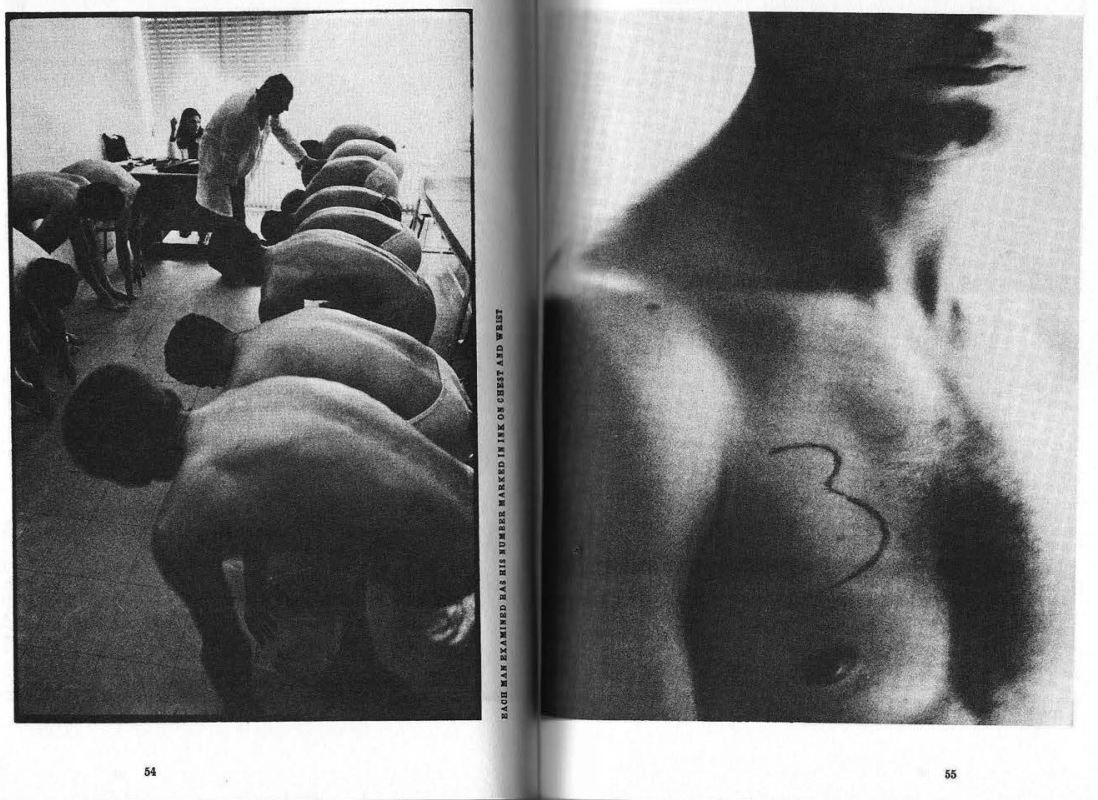

Abb. 1: Jean Mohr (Fotografie) und John Berger (Text), Medical Examination, Istanbul, in: A Seventh Man (1975), London 2010, S. 54-55. 


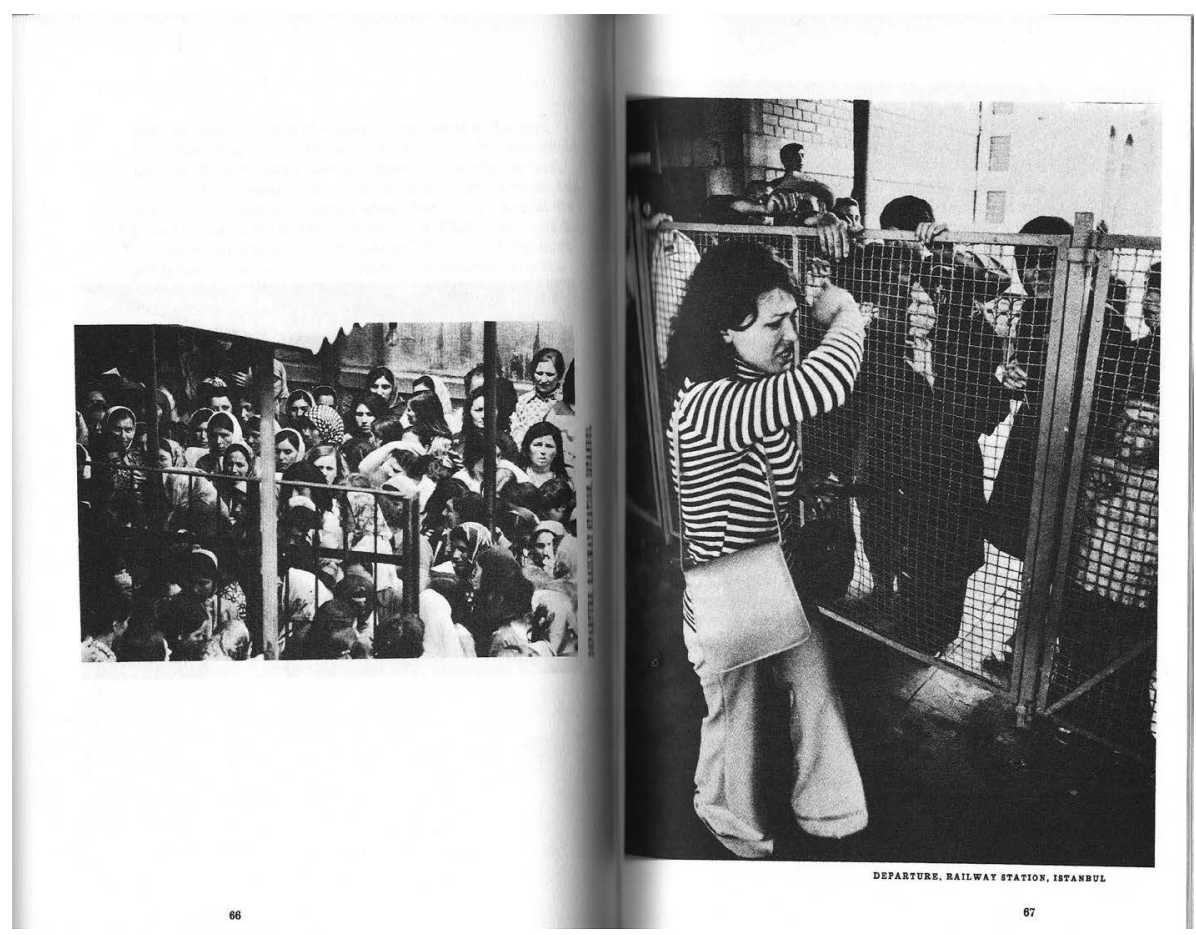

Abb. 2: Jean Mohr (Fotografie) und John Berger (Text), Departure, Railway Station, Istanbul, in: A Seventh Man (1975), London 2010, S. 66-67.

Flughäfen, Bahnhöfe, Wartesäle sind Orte, an denen sich nur schwerlich individuelle Spuren ablagern können. Sie sind Ausdruck einer transitorischen Existenz, für viele Ausgang oder Ziel einer Reise, Orte des Übergangs, die nicht für einen dauerhaften Aufenthalt geeignet sind. Nicht umsonst konzentriert sich das Thema der frühen Arbeitsmigration nach Deutschland beharrlich in Bildern der Hauptbahnhöfe, an denen viele Einwanderer erstmals bundesdeutschen Boden betraten. Die Sonderzüge mit „Gastarbeiter*innen“ aus Italien, Griechenland, der Türkei und Jugoslawien endeten in den 1960er Jahren auf Gleis 11 des Münchner Hauptbahnhofs, von wo sie zu ihren Arbeitgebern im gesamten Bundesgebiet weiter verteilt wurden (Terkessidis, 2000, 19). ${ }^{1}$ Zeitgenössische Fotografien zeigen die erschöpften, ängstlichen oder hoffnungsfrohen Menschen, die gerade den Zügen entstiegen waren (Abb. 3). Marc Augé tituliert Bahnhöfe als „Nicht-Orte“ (Non-Lieux), an denen sich keine relationale Verbundenheit von Menschen ausprägen kann. An diesen Nicht-Orten treffen sich

1 Zur türkischen Arbeitsmigration nach Deutschland vgl. Eryılmaz, Jamin, 1998, S. 140-143 mit einer Bildstrecke von Selahattin Kaya/DoMIT-Archiv zur Ankunft im Deutschland der 1960er Jahre. Zu sehen sind Migranten am Münchner Hauptbahnhof, Gleis 11, und im Warteraum des Bunkers in der „Weiterleitungsstelle“. Vgl. auch die verschiedenen privaten Ankunftsfotografien von Migrant ${ }^{\star}$ Innen in Projekt Migration, 2005. 
die Wege vieler Menschen, ohne dass sie unweigerlich tatsächlich in Beziehung zueinander stehen. Augé nennt die Nicht-Orte

das Maß unserer Zeit, ein Maß, das sich quantifizieren lässt und das man nehmen könnte, indem man - mit gewissen Umrechnungen zwischen Fläche, Volumen und Abstand - die Summe bildete aus den mobilen Behausungen, die man als ,Verkehrsmittel' bezeichnet (Flugzeuge, Eisenbahnen, Automobile), den Flughäfen, Bahnhöfen und Raumstationen, den großen Hotelketten, den Freizeitparks, den Einkaufszentren ... (Augé, 2012, 84).

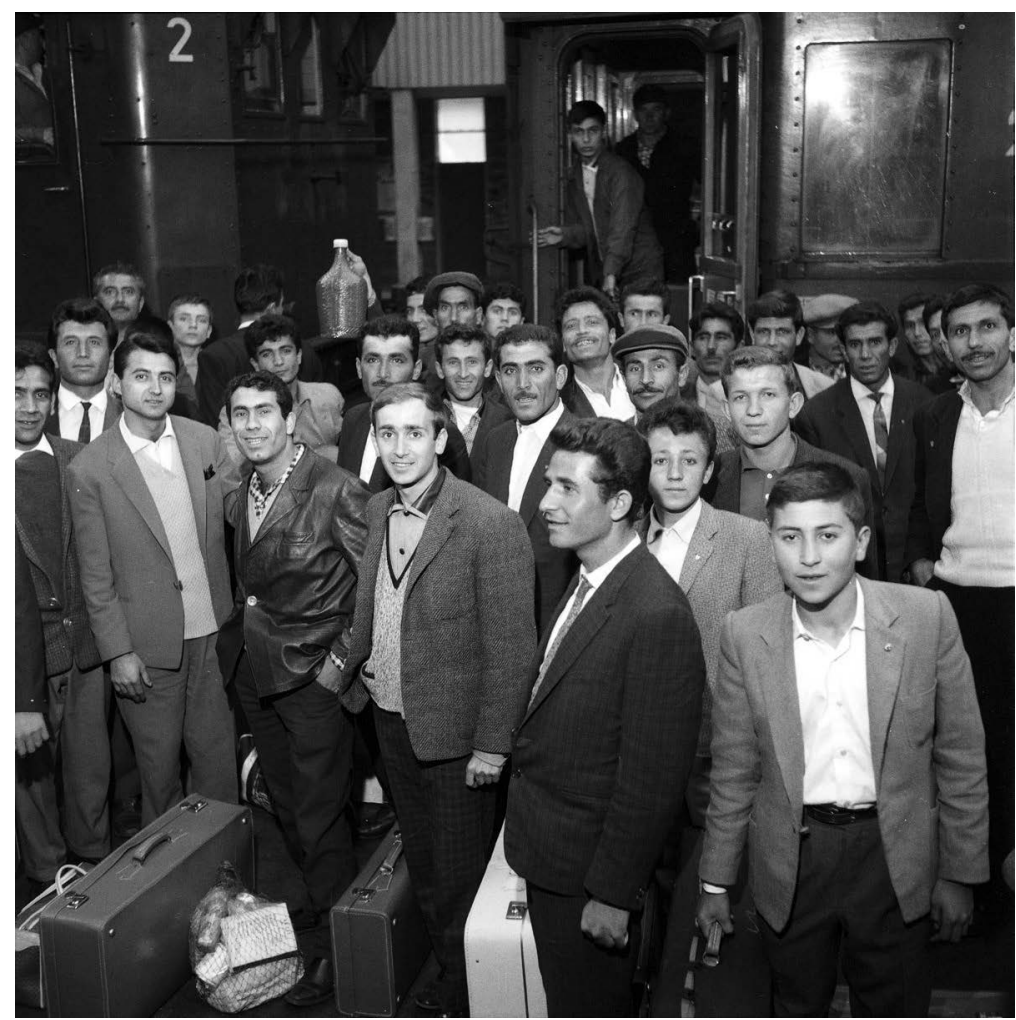

Abb. 3: Felicitas Timpe, Hauptbahnhof München, Ankunft türkischer Gastarbeiter, 1964, Bayerische Staatsbibliothek München, Bildarchiv, Sign. timp-016732.

Obgleich sich moderne Transportmittel und ihre Ankunfts- oder Abfahrtsorte eigentlich wenig mit Begriffen wie Heim oder Heimat assoziieren lassen, sind insbesondere Bahnhöfe nicht selten Anziehungspunkte für Marginalisierte oder Minoritäten, darunter auch Kumulationsorte für Migrant ${ }^{*}$ innen, wie beispielsweise Aufnahmen von Candida Höfer aus Köln und Düsseldorf der 1970er Jahre zeigen. Der deutschtürkische Schriftsteller Aras Ören beschreibt in seinem Gedicht Bahnhoflar, 
eine Adaption des deutschen „Bahnhofs“ ins Türkische, die zentrale soziale Bedeutung dieses Ortes: „Wo sind/auf Bahnhöfen Pauken und Trompeten? / Sie spielen nicht / sind nicht da. / Oberflächlich gesehen: Landsleute umarmen, / von Einkauf und Teuerung, von Lohn und Verdienst / zu reden, / kommt man hin.“ (Ören, 1980, 2)2 In Höfers Fotografien sind eben diese Bahnhofsviertel als klassische Ankunftsorte der Migration immer wieder im Blick, wenn sie „Gastarbeiter*innen“ beim Ausharren auf öffentlichen Sitzgelegenheiten oder im Gespräch auf der Straße mit der Kamera ins Bild holte. Höfer visualisiert die Männer aus größerer Distanz, um sie als Gruppe zu erfassen. Sie reden miteinander, einige haben Plastiktüten in den Händen, als seien sie nur kurz von einem Einkauf gekommen, andere blicken auf die Uhr. Hier dehnt sich die Zeit, und das Warten ist zugleich eine Freizeitbeschäftigung wie auch eine Möglichkeit der sozialen Kommunikation. Der Schriftsteller John Berger schreibt über den Anziehungspunkt Hauptbahnhof in seinem Buch A Seventh Man:

Arbeitsemigranten, die bereits in der Metropole leben, haben die Gewohnheit den Hauptbahnhof aufzusuchen. Um beieinanderzustehen und zu reden, die einlaufenden Züge zu beobachten, die neuesten Nachrichten aus der Heimat zu erfahren, den Tag zu erwarten, da sie die Rückreise antreten werden. (Berger, 1976, 64).

Das Warten kontrastiert zu den Reisewegen, die hinter diesen Menschen liegen. Als „Gastarbeiter*innen“ mussten sie erst viele Tausend Kilometer und Asien hinter sich lassen, um in ihrer neuen europäischen Heimat zu Wartenden zu werden.

Im Bild des Bahnhofs manifestiert sich aber auch die transitorische Existenz der nach Deutschland importierten Arbeiter*innen, deren Verweildauer von Anbeginn auf eine unbestimmte Dauer angelegt war. Mit dem Bild des Bahnhofs und der Koffer ist zudem verbunden, dass die Betreffenden niemals richtig an ihrem Zielort ankommen, ist doch der Bahnhof ein Umschlagplatz für Reisende, die nie lange dort verweilen: Seit dem Jahr 1955 schloss die Bundesrepublik Anwerbeabkommen mit verschiedenen Ländern, darunter Italien, Griechenland, Spanien, Portugal und mit der Türkei, um dem prosperierenden deutschen Arbeitsmarkt temporär Arbeitskräfte zuzuführen (Dietert, 2010, 162-164). Bis zum Anwerbestopp 1973 kamen in der Folge mehrere hunderttausende „Gastarbeiter*innen“, deren in der Öffentlichkeit etablierte Bezeichnung ${ }^{3}$ bereits festlegte, dass diese nicht als Bleibende sondern nur als

2 In Eryllmaz, Jamin, 1998, S. 237, finden sich Fotografien von Erika Sulzer-Kleinemeier, die türkische Einwanderer am Frankfurt Bahnhof zeigen: „Bahnhöfe in den Städten waren anfangs Haupttreffpunkte ausländischer Arbeitsnehmer in der Freizeit. Hier traf man Landsleute aus verschiedenen Betrieben. Im Wohnheim war dazu nur wenig Platz."

3 Siehe dazu die umfangreiche Dokumentation in Ausst.-Kat. Berlin 2009, S. 279-285. Eine frühe Kritik am Begriff „Gastarbeiter“ in Nachfolge zum Terminus „Fremdarbeiter“ äußert bereits Delgado, 1972, S. 9. 
zeitweilig in Deutschland lebende Personen wahrgenommen wurden. Der Terminus „Gastarbeiter*innen“ wurde zwar nicht im amtlichen Sprachgebrauch verwendet, war jedoch bereits seit Beginn der Arbeitsmigration nach Deutschland in der Öffentlichkeit etabliert. Beispielhaft ist die Verwendung des Begriffs in den deutschen Medien, so am 26. März 1960 in der Münchner Illustrierten und am 7. Oktober 1964 in der Zeitschrift Der Spiegel, die mit der Titelgeschichte Gastarbeiter in Deutschland warb. John Berger beschrieb die Bedeutung dieses ephemeren Aufenthaltsstatus, die prekären Lebensbedingungen und psychischen Strapazen der Migranten in den 1970er Jahren:

Was diese Emigration von anderen in der Vergangenheit unterscheidet, ist die zeitliche Begrenzung. Nur den wenigsten Arbeitern wird gestattet, in dem Land, in das sie gekommen sind, ansässig zu werden. Ihre Arbeitsverträge gelten in der Regel für ein Jahr oder höchstens zwei. [...] Nicht Menschen wandern ein, sondern Maschinenpfleger, Bodenfeger, Schaufelschwinger, Zementmixer, Putzer, Bohrer usw. Dies ist die Bedeutung der temporären Emigration. Um wieder ein Mensch (Ehemann, Vater, Bürger, Patriot) zu werden, muß der Emigrant in die Heimat zurückkehren. Die Heimat, die er verließ, weil sie ihm keine Zukunft bot. (Berger, 1976, 58).

\section{Unendliche Aufbrüche: Arbeitsmigrant ${ }^{*}$ inenn als daverhaft Reisende}

Der unsichere Aufenthaltsstatus der „Gastarbeiter*innen“ in Deutschland findet seine Korrespondenz in bildlichen Formulierungen, die die Eingewanderten als Fremde konnotieren. Gruppenbildungen, andere Kleidungen, Physiognomien und Körperhaltungen wurden seit den 1960er Jahren immer wieder in Presseaufnahmen verbildlicht. Sie zeigen Menschen, die sich scheinbar nicht ihrer Umwelt anpassen, sich anders verhalten als die native Bevölkerung ihres „Gastlandes“. Hartnäckig hielt sich jedoch ein Bildvokabular, das den Aufenthalt der Migrant*innen zur andauernden Reise jenseits einer tatsächlichen Ankunft in der Mitte der Gesellschaft erhob. So wurde der Artikel In vier Jahren droht eine türkische Einwanderungswelle von Wolfgang Philipp (Frankfurter Allgemeine Zeitung, 2.2. 1983, Nr. 27, 9) illustriert von einer Fotografie der Bildjournalistin Barbara Klemm, die Männer mit Koffern am Bahnhof zeigen (Abb. 4). Die Bildunterschrift klärt über die Umstände auf: „Gastarbeiter auf dem Weg in den Urlaub - die meisten kommen zurück“. Klemm stellte sich mitten in den Trubel und fotografierte den Menschenauflauf aus nächster Nähe. Koffer und Menschen stehen dichtgedrängt am Bahnsteig. Die Blicke sind nach links gerichtet, vermutlich fixieren sie den eintreffenden Zug. Klemms Aufnahme korrespondiert mit den Fotografien, die „Gastarbeiter*innen“ beim Aufbruch aus ihren Heimatländern oder bei der Ankunft in ihren Zielländern zeigen. Somit sind die regelmäßigen Ferienreisen der Migrantinnen 
in Deutschland nur Teil einer andauernden Ankunft und Abfahrt, die zum Grundmotiv der Arbeitsmigration schlechthin wird. Diese Bildfindungen haben ihre Analogie im Selbstverständnis der Bundesrepublik als explizit kein Einwanderungsland: Im Jahr 1977 erklärte die Bundesregierung auf Grundlage eines Kommissionsberichtes, dass Deutschland kein Einwanderungsland sei, eine Position, die über Jahre beibehalten wurde und im Widerspruch zu den gleichzeitigen Maßnahmen zur Integration von Immigranten stand. (D’Amato, 2007, 22). Auch in den Koalitionsvereinbarungen des linkskonservativen Bündnisses unter Helmut Kohl wurde in den achtziger Jahren festgeschrieben: „Deutschland ist kein Einwanderungsland.“(Herbert, 2003, 249-250). Diese Perspektive negierte jedoch, dass aus den temporär ins Land geholten Arbeitern längst tatsächliche Einwanderer geworden waren. Obgleich die Immigration neuer „Gastarbeiter*innen“ in den 1970er Jahren stagnierte, führte der Familiennachzug und der bei vielen Migrant*innen nun langjährige Aufenthalt in Deutschland zu einer Verfestigung des Aufenthaltsstatus. (vgl. Oltmer, 2013, 54). Doch in den 1980er Jahren wurden neue politische und gesetzliche Wege beschritten, um die sesshaft gewordenen Ausländer zur Rückkehr in ihre Herkunftsländer zu motivieren. Die bereits von der sozialliberalen Koalition beschlossenen Maßnahmen wurden nach dem Regierungswechsel unter der Bundeskanzlerschaft von Helmut Kohl 1983 als „Gesetz zur Förderung der Rückkehrbereitschaft von Ausländern“ umgesetzt. Dieses sah für ausländische Arbeitnehmer eine Abfindung („Rückkehrprämie“) und eine vorzeitige Erstattung von Arbeitnehmerbeiträgen aus der Rentenversicherung vor (vgl. Yano, 2000, 7; Eryllmaz, 2011, 39) - unter der Voraussetzung, dass sie das Land binnen vier Wochen verlassen sollten. Eine Rückwanderungswelle war die Folge, und Fotograf*innen wie Manfred Vollmer oder Brigitte Kraemer begleiteten den Auszug der Migrant*innen. Beide widmeten sich der Siedlung Duisburg-Hüttenheim, die besonders vom Wegzug der ausländischen Arbeiter ${ }^{\star}$ innen betroffen war. Diese hatten die hohen Rückkehrprämien des zentralen Arbeitgebers Mannesmann angenommen: die Firma bot den ausländischen Arbeitern nicht nur zwei zusätzliche Monatslöhne, wenn sie nicht erst bis zum 30. Juni 1984 unterschrieben (dem letzten Tag, an dem das Gesetz wirksam sein sollte), sondern bereits im Januar ihre Arbeitsplätze aufgaben. Besonderer Druck wurde durch betriebsinterne Sprachtests aufgebaut, die vorgeblich dazu dienen sollten, den Reüssierenden eine höher qualifizierte Tätigkeit anzubieten. Doch zugleich fürchteten jene, die den Test nicht bestanden, die Arbeit zu verlieren (Hunn, 2005, 480-482). So wuchs der Druck, die Rückkehrprämie vorzeitig anzunehmen. Den durch das Bundesgesetz und die Firmenstrategie zum Abbau von Personal herbeigeführten Exodus aus Duisburg-Hüttenheim fotografierten Vollmer und Kraemer, als sie im Jahr 1984 türkische Familien kurz vor ihrer Abreise in leergeräumten Wohnungen besuchten (Abb. in At Home, 2012, 42, 43, 163). Nur noch die Tapeten erzählen von einem abgeschlossenen Lebensabschnitt. Vollmers Fotografie 
mit dem Titel „Exodus in Hüttenheim“ (1984) zeigt ein Kind in Gummistiefeln und Winterjacke, das verloren an der Wand einer leeren Wohnung lehnt. Die Aufnahme thematisiert das Alleinsein, zugleich ist sie Ausweis einer rücksichtslosen Ausländerpolitik. Vollmer begleitete die Familie noch auf der Straße, als sie mit ihren Koffern in den Händen ihr Viertel verlassen (Abb. 5, siehe auch Vollmer, 2012, 120). Die am Wegrand liegenden Möbel sind Reste der ausgeräumten Wohnungen; die Dinge verweisen auf das Schicksal, ästhetische Vorlieben und Wohnkultur ihrer vormaligen Besitzer. Bei Kraemer sind den vier Frauen mit ihren Kindern nur noch zwei Matratzen geblieben. Ernst blickt eine von ihnen mit weit geöffnetem Mund in die Kamera, während die ältere Frau auf der linken Seite in einer Geste der Betroffenheit ihren Kopf auf die Hand stützt. Die räumliche Distanz zwischen der Fotografin und ihren Modellen ist Ausdruck für die Unmöglichkeit, hier einen wirklichen Beistand zu geben. Die Fotografierten sind letztlich allein mit ihrem Schicksal, das sie kaum ertragen oder meistern können (vgl. Vogt, 2012, 61). Die sensiblen, empathischen Fotografien dieser von der Bundesregierung und den Unternehmen erkauften Abreise korrespondieren zu den Ankunftsbildern der frühen 1970er Jahre und lassen gemeinsam ein Narrativ entstehen, das Migration als andauernde Reise versteht, die keine endgültige Ankunft zulässt.

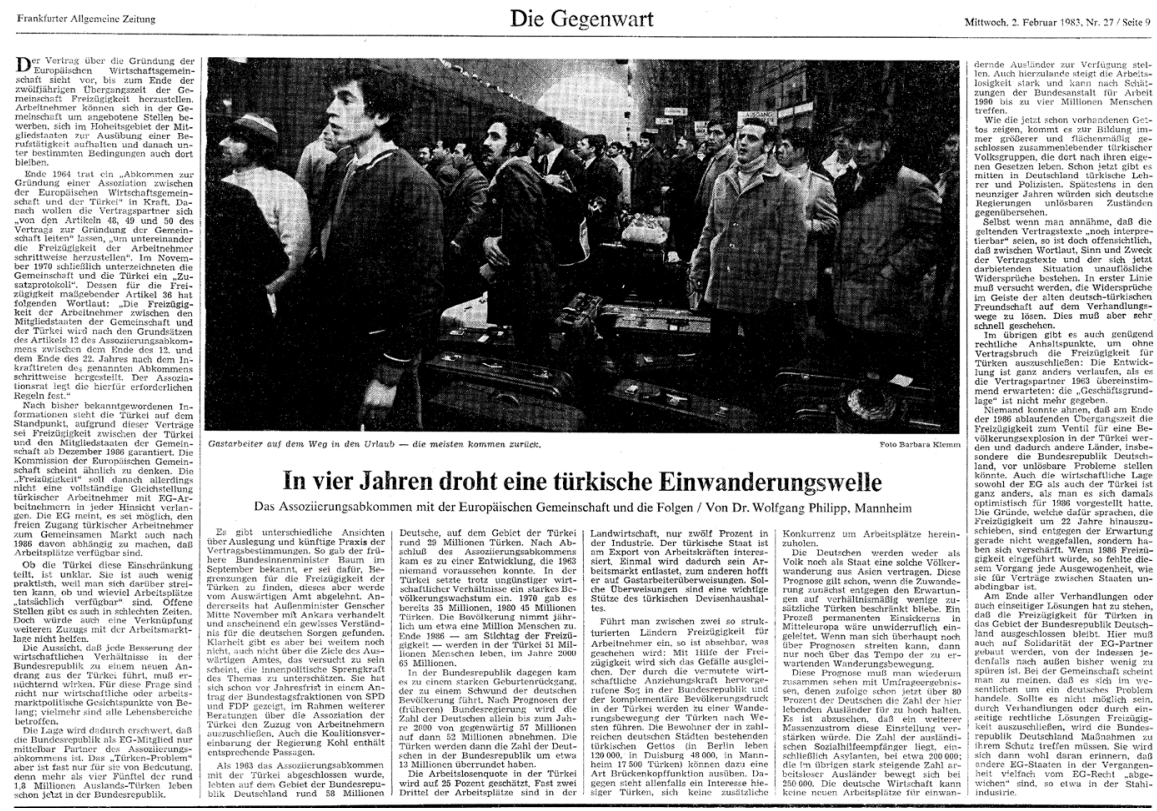

Abb. 4: Barbara Klemm (Fotografie) und Wolfgang Philipp (Text), In vier Jahren droht eine türkische Einwanderungswelle, in: Frankfurter Allgemeine Zeitung, Nr. 27, 2. 2. 1983, S. 9. 


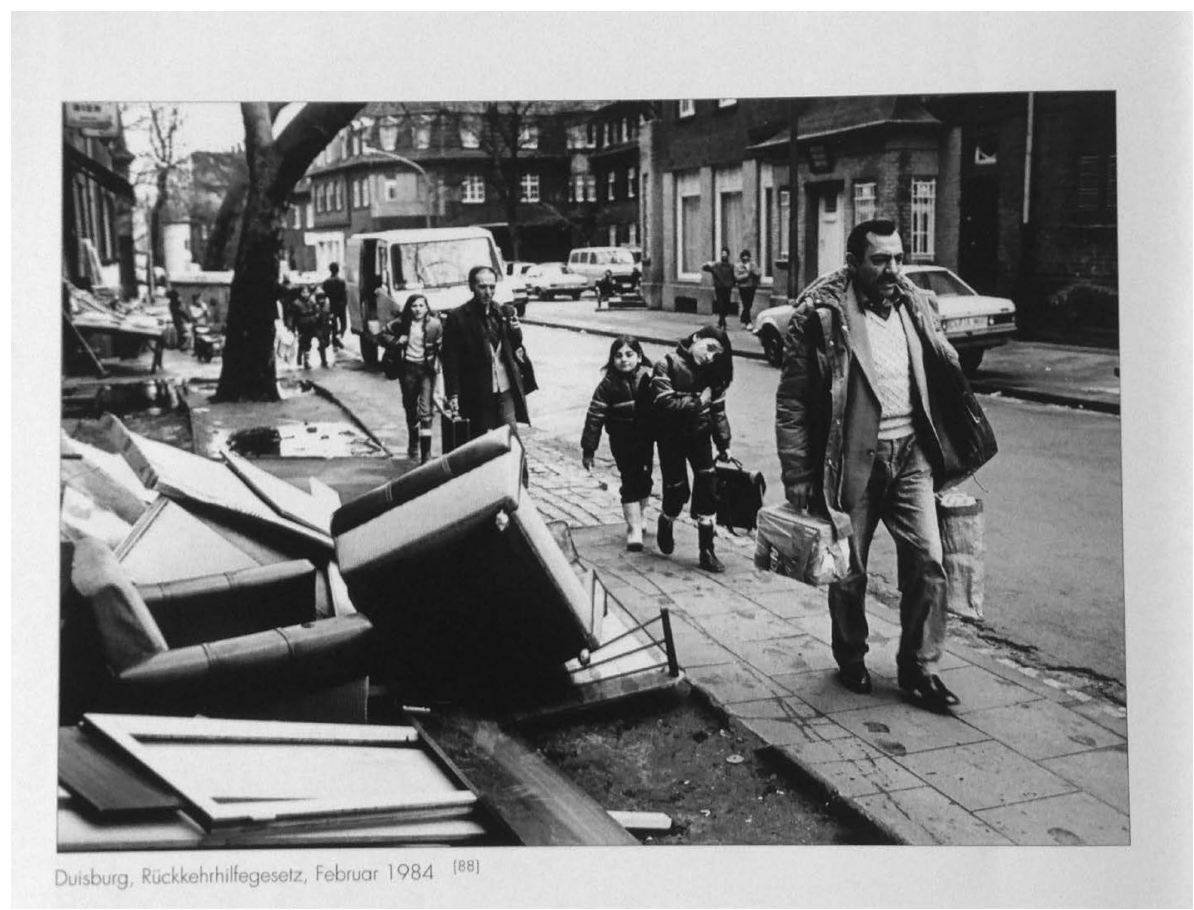

Abb. 5: Manfred Vollmer, Duisburg, Rückkehrhilfegesetz, Februar 1984, in: Vollmer, M., Mein Revier. Ein Vierteljahrhundert im Bild. Das Ruhrgebiet von 1965 bis 1989, Essen 2012, S. 120.

\section{Von überfüllten Archen: Migration und Flucht im Bild der Schiffspassage}

Dass Europa von Geflüchteten überflutet wird, sie an den Außengrenzen stranden und den Kontinent wie eine Lawine überrollen, gehört zum inzwischen etablierten und durchaus kritisierten öffentlichen Sprachgebrauch. Medial verbreitete Bilder von überfüllten Schiffen und verzweifelten Gekenterten begleiten diese textlichen Zuschreibungen. Sie betonen, dass Geflüchtete nicht nur im Überfluss zu „uns“ kommen, sondern auch überflüssig sind. Interessant ist aber, dass überfüllte Boote und verirrte Archen auch dann zur visuellen Beschreibung von Flucht, Asyl und Migration eingesetzt werden, wenn Auswanderung oder Flucht gar nicht auf Wasserwegen von statten gehen. Beispielhaft sind Karikaturen oder Titelbilder deutscher Magazine und rechtsextreme Propagandaplakate aus den frühen 1990er Jahren, die sich der Rhetorik „Das Boot ist voll“ bedienen und damit auf die 
Arbeitsmigration jener Jahre abzielen. ${ }^{4}$ Gerade Migranten und Asylsuchende aus Ost-, Ostmittel- und Südosteuropa, die ab Ende der 1980er Jahren einen großen Teil der Asylantragsteller stellten, kamen über Land. Dennoch wurde ihr Dasein als dauerhafte grenzverletzende Seefahrt visualisiert.

Obgleich die wenigsten Arbeitsmigrant*innen tatsächlich mit dem Schiff in die Bundesrepublik gekommen sein dürften, findet sich im medial verbreiteten Bild der überfüllten Arche bereits in den 1970er Jahren ein schlagkräftiges Zerrbild von Einwanderung. Unweigerlich verbunden mit diesen Bildern für Flucht, Migration und Asyl war die Furcht vor einer apokalyptischen Überschwemmung Deutschlands und Überschwemmung in Gestalt flüchtender Menschen. Diese bildlichen Formulierungen für Migration und Flucht schüren Ängste, indem sie auf einem archaischen Bildvokabular basieren, dass auf christlichen und antiken Vorbildungen basiert. Die biblische Arche wie auch die Irrfahrten des Odysseus stehen für ein zunächst zielloses und planloses Fahren. Wenn die Arche auf die Gefahr der katastrophalen Sintflut reagiert, so muss Odysseus einerseits den Gefahren des Meeres und seiner Gestalten (Sirenen) trotzen, andererseits ist er ein Kriegsherr, der selbst Unglück und Tod bringt. Die Kontinuität medialer Visualisierungen der historischen Arbeitsmigration und der heutigen Fluchtbewegungen im Bild des überfüllten Schiffes zeigt aufschlussreich, dass nicht unbedingt ein reales Reisemittel abgebildet werden muss, sondern auf einer Metaebene Ängste und Gefahren heraufbeschworen werden.

Auf die gefährlichen Überwasserfahrten der Geflüchteten vom afrikanischen Kontinent reagieren bildende Künstler, indem sie reale Erfahrungen und deren nachrichtliche Überlieferungen in komplexe künstlerische Arbeiten übersetzen. Denn statt die überfüllten Schiffe zu einfachen Zeichen der Flucht zu erheben, wie es viel zu häufig in vielen Medienbildern geschieht, finden Künstlerinnen wie Dieter Huber oder Kader Attia poetische und politische künstlerische Translationen für die Routen und Passagen der Geflüchteten. ${ }^{5}$ Beide verwenden ähnliche Titel für ihre Arbeiten: Hubers Mare Morto (2011) und Attias La Mer Morte (2015) umkreisen das todbringende Mittelmeer, das zugleich Ort der Überfahrt wie auch ein Grab für zahlreiche Geflüchtete geworden ist. Beide widmet sich den Spuren dieser Flucht mit tragischem Ausgang. Während in Attias Installation gebrauchte Kleidungsstücke auf dem Boden verteilt sind und die Assoziation an angeschwemmte Textilien Ertrunkener evozieren, ist Hubers Mare Morto ein reines Fotoprojekt. Es ist in drei Kapitel mit jeweils 33 Fotografien unterteilt: Mare Morto

4 Vgl. die Beispiele bei Oltmer, 2015, S. 115-117.

5 Im Katalog zur Ausstellung Streamlines (Kat. Hamburg 2016) finden sich zahlreiche künstlerische Projekte, die sich mit Menschen- und Warenströmen über Wasser beschäftigen. 
zeigt zerstörte Boote von Geflüchteten (Abb. 6), Refugees widmet sich individuellen Fund- und Beweisstücken der Flucht, während Stranded gestrandete Schiffe ins Bild holt, die teilweise repariert und erneuert wurden. Die aufgetürmten Wracks mit den ineinander verkeilten Relikten - Holz, Plastik und Textilien - steht stellvertretend für die zerstörten Hoffnungen und Träume der Menschen, aus denen Müll, Zerstörtes und Vergessenes wird. Auf den süditalienischen Inseln, die Huber besuchte, fand er zahlreiche dieser Spuren. Wasser, Boote und Textilien verbinden alle drei Kapitel des Zyklus. Dabei stehen die Funde und Relikte auch ein für die verdrängten Ereignisse, denn bereits seit Jahrzehnten versuchen Menschen, ihre Heimaten über das Mittelmeer zu verlassen. Die Schriftstellerin Maxi Obexer nahm sich in ihrem Theaterstück Das Geisterschiff (2007) eines Schiffsunglücks von Weihnachten 1996 an, als 283 Geflüchtete vor der Südküste ertranken. Obwohl Fischer nach Wochen Leichenteile in ihren Netzen fanden, ignorierte die Küstenwache den Vorfall - die Körper wurden ins Meer zurückgeworfen. Erst nach Jahren wurde die Katastrophe publik und wurde dennoch von der italienischen Regierung geleugnet. Obexers Stück weist in eine Zeit zurück, als Grenzübertritte über Wasser noch nicht auf der Agenda der Politik und der Medien standen. Erst die sogenannte Flüchtlingskrise vom Spätsommer 2015 rückte mit einem Schlag eine längst etablierte Fluchtstrecke in den Blick. So lässt sich von einer verspäteten Erfahrung im Bewusstsein der Öffentlichkeit sprechen und eine vorgeblich neue Krise, die keine ist. Auch Hubers Fotoprojekt entstand bereits 2011 und zeigt Motive, die sich ohne Weiteres auch auf gegenwärtige Ereignisse wie etwa den Untergang eines Schiffs mit 700 Geflüchteten im April 2015 oder mit 400 vermuteten Toten im April 2016 beziehen lassen. ${ }^{6}$ Huber wählte für seine Aufnahmen die Form des Rundbildes; der kreisrunde Ausschnitt verweist auf das Bullauge wie auf das Fernrohr und damit auf das Sehen und Zeigen. ${ }^{7}$ Damit verweist Huber mit den Mitteln der Kunst auf das Übersehene, Ignorierte und Vergessene. Er macht die Spuren der Migrationsrouten an ihren Tatorten sichtbar und entreißt sie damit dem Lauf der Zeit. Im einem metaphorischen Sinne bläst er den Sand von den Planken, Kleidern und Schiffskörpern, der diese Relikte zu überdecken droht.

6 http://www.sueddeutsche.de/news/politik/migration-bis-zu-700-tote-fluechtlinge-nachschiffsunglueck-befuerchtet-dpa.urn-newsml-dpa-com-20090101-150419-99-03483; http://www. br.de/nachrichten/mittelmeer-unglueck-fluechtlinge-100.html [7. 7. 2016].

7 Die Referenz auf das Bullauge und Fernrohr formuliert Dieter Huber in seinem Vorwort zu seinem Buch Mare Morto (Huber, 2011, 4). 


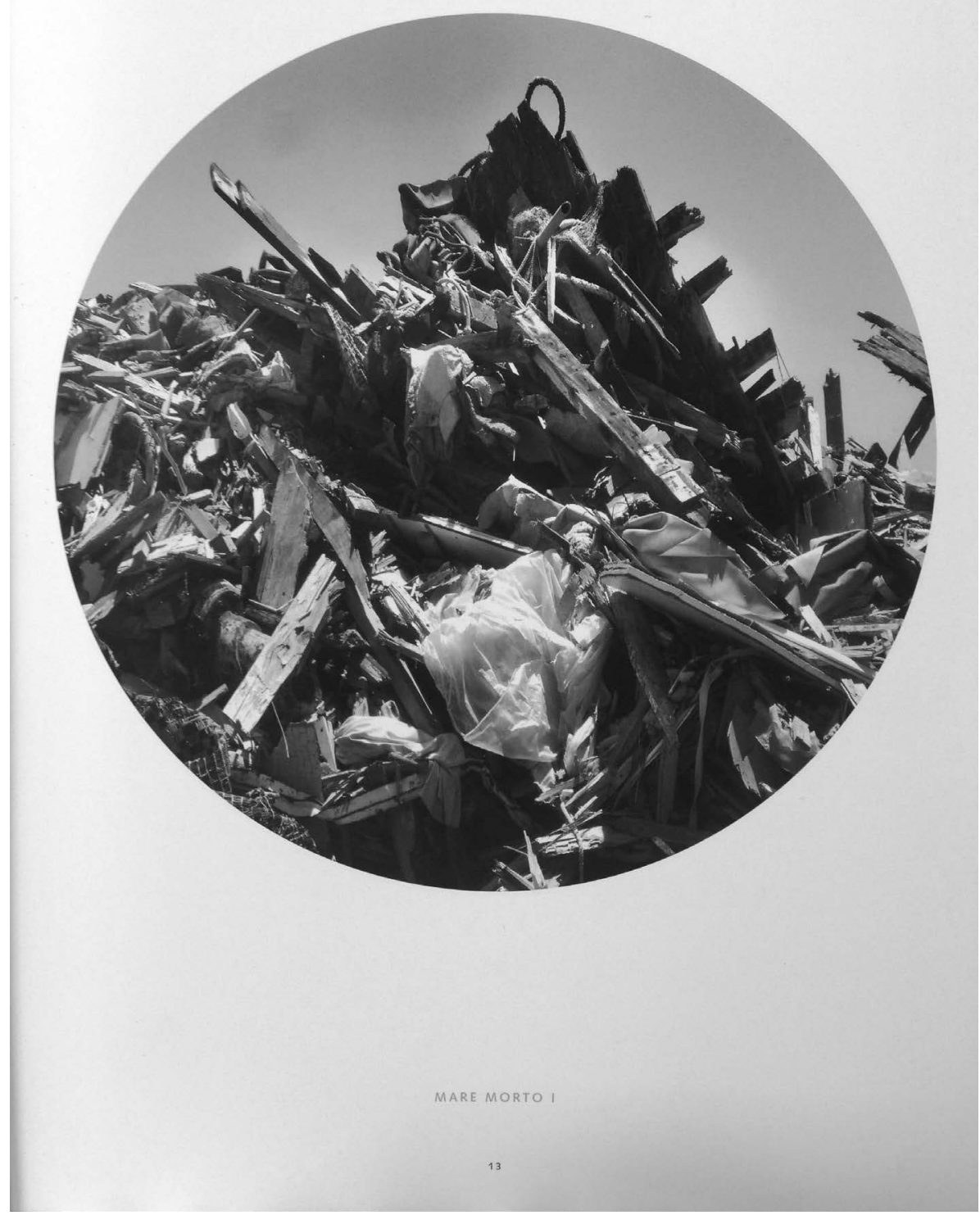

Abb. 6: Dieter Huber, Mare Morto I, in: Huber, D., Mare Morto. Refugees. Stranded. Bielefeld 2011, S. 13. 


\section{Bibliografie}

At Home. Der Blick durchs Schlüsselloch. Wohnen im Ruhrgebiet - gesehen durch die

Kunst (Hg. Dunkmann, N., Vogt, C.), Oberhausen 2012.

Augé, M., Nicht-Orte (1992), München ${ }^{3} 2012$.

Berger, J. et al., A Seventh Man, London 1975.

Berger, J. et al., Arbeitsemigranten. Erfahrungen/Bilder/Analysen, Reinbek bei Hamburg 1976.

D’Amato, G., Die politisch-rechtlichen Bedingungen, in: Interkulturelle Literatur in Deutschland. Ein Handbuch (Hg. Chiellino, C.), Stuttgart 2007 (2. Aufl.), S. 18-34.

Dietert, A., Einwanderung aus der Türkei nach Hamburg, in: Begegnungen - Ilişkiler. Hamburg und die Türkei in Geschichte und Gegenwart (Hg. Dawletschin-Linder, C., Dietert, A.), Hamburg 2010, S. 156-223.

Fremde Heimat. Eine Geschichte der Einwanderung aus der Türkei (Hg. Eryılmaz, A. et al.), Essen 1998.

Eryılmaz, A. et al., 50 Jahre Migration aus der Türkei. Ein historischer Rückblick, in: Geteilte Heimat. 50 Jahre Migration aus der Türkei. Paylaşılan Yurt (Hg. Eryılmaz, A., Lissner, C.), Essen 2011, S. 34-43.

Herbert, U., Geschichte der Ausländerpolitik in Deutschland. Saisonarbeiter, Zwangsarbeiter, Gastarbeiter, Flüchtlinge, Bonn 2003.

Huber, D., Mare Morto. Refugees. Stranded, Bielefeld 2011.

Hunn, K., „Nächstes Jahr kehren wir zurück..." Die Geschichte der türkischen „Gastarbeiter“ in der Bundesrepublik, Göttingen 2005.

Oltmer, J., Migration im 19. und 20. Jahrhundert, München 2013 (2. Aufl.).

Oltmer, J., Politisch verfolgt? Asylrecht und Flüchtlingsaufnahme in der Bundesrepublik, in: Immer bunter. Einwanderungsland Deutschland (Hg. Stiftung Haus der Geschichte der Bundesrepublik Deutschland), Bonn 2015, S. 107-123.

Ören, A., Bahnhoflar - Bahnhöfe, in: Anadil. Yurtdışındaki türklerin yazın ve sanat dergisi 1/1, 1980, S. 2.

Projekt Migration (Hg. Eryılmaz, A.), Köln 2005.

Streamlines. Ozeane, Welthandel und Migration, Hamburg 2016.

Terkessidis, M., Migranten, Hamburg 2000.

Vogt, C., Heimat liegt im Auge des Betrachters. Wohngefühle im Ruhrgebiet, in: At Home. Der Blick durchs Schlüsselloch. Wohnen im Ruhrgebiet - gesehen durch die Kunst (Hg. Dunkmann, N., Vogt, C.), Bielefeld 2012, S. 59-66. 
Vollmer, M., Mein Revier. Ein Vierteljahrhundert im Bild. Das Ruhrgebiet von 1965 bis 1989, Essen 2012.

Yano, H., Migrationsgeschichte, in: Interkulturelle Literatur in Deutschland. Ein Handbuch (Hg. Chiellino, C.), Stuttgart, Weimar 2007 (2. Aufl.), S. 1-17. 


\section{Burcu Dogramaci}

\section{Prišli, da ne bi ostali: podobe prihoda kot vizualne reprezentacije migracij}

Ključne besede: migracije, podobe prihodov in odhodov, gostujoči delavec, emigracija/imigracija, fotografija

Podobe odhodov in prihodov so tesno povezane z imigracijo in emigracijo. Številne zgodovinske fotografije na primer prikazujejo turške "gastarbajterje«, ki v šestdesetih letih 20. stoletja zapuščajo Istanbul in prihajajo v München. Te dokumentarne fotografije zaznamujejo železniške postaje, vlaki, ljudje s kovčki, nered, radovednost in strah. Podobe prenatrpanih železniških postaj so krožile v medijih in migracije prevajale $\mathrm{v}$ popotovanje brez prihoda $\mathrm{v}$ ciljnih deželah - reprezentativni simbol je postal kovček, pogosto razstavljen na razstavah o migracijah. Ekvivalent tem podobam prihoda so bile v osemdesetih letih preteklega stoletja fotografije turških "gostujočih delavcev«, ki zapuščajo Nemčijo, ko so jim izplačali visoke denarne nagrade, če so zapustili deželo. Fotografi, kot na primer Brigitte Kraemer in Manfred Vollmer, so spremljali družine med odhodom od doma. Članek zgodovinske fotografije primerja s sodobnimi medijskimi reprezentacijami prihajajočih beguncev ter se sprašuje o razlikah in podobnostih. Ali obstaja podobje, ki obsega različna tranzitivna stanja imigracij? Kakšna pripisovanja formulirajo te slike? Ali lahko umetnost proizvede nasprotne podobe? 


\section{Burcu Dogramaci}

\section{Coming, but not Staying: Pictures of Arrivals as Visual Representations of Migration}

Keywords: migration, images of arrivals and departures, guest workers, emigration/immigration, photography

Images of departure and arrival are closely linked to immigration and emigration. Numerous historical photographs show, for example, Turkish "guest workers" leaving Istanbul and arriving in Munich in the 1960s. Railway stations, trains, people with suitcases, disorder, curiosity and fear characterize these documentary photographs. Images of crowded train stations circulated in the media and translated migration as a journey without arrival in the target countries - the journey finding its representative symbol in the suitcase, often exhibited in migratory exhibitions. Counterparts to these arrival images were photographs from the 1980s, as Turkish "guest workers" received high return premiums when they left Germany. Photographers such as Brigitte Kraemer or Manfred Vollmer accompanied families during their exodus from their homes. This essay compares the historical photographs with contemporary media representations of arriving refugees and asks about differences and analogies: Is there an overarching imagery for the transitive state of immigration? What formulates the shots? And is art able to produce counter images? 